Article

\title{
A Family of the $r$-Associated Stirling Numbers of the Second Kind and Generalized Bernoulli Polynomials
}

\author{
Paolo Emilio Ricci ${ }^{1, *(\mathbb{D}}$, Rekha Srivastava ${ }^{2}$ (D) and Pierpaolo Natalini ${ }^{3}$ \\ 1 Dipartimento di Matematica, International Telematic University UniNettuno, 39 Corso Vittorio Emanuele II, \\ I-00186 Rome, Italy \\ 2 Department of Mathematics and Statistics, University of Victoria, Victoria, BC V8W 3R4, Canada; \\ rekhas@math.uvic.ca \\ 3 Department of Mathematics and Physics, Roma Tre University, Largo San Leonardo Murialdo, 1, \\ I-00146 Rome, Italy; natalini@mat.uniroma3.it \\ * Correspondence: p.ricci@uninettunouniversity.net
}

Citation: Ricci, P.E.; Srivastava, R.;

Natalini, P. A Family of the

$r$-Associated Stirling Numbers of the Second Kind and Generalized Bernoulli Polynomials. Axioms 2021, 10, 219. https://doi.org/10.3390/ axioms10030219

Academic Editor: Palle E.T. Jorgensen

Received: 17 August 2021

Accepted: 3 September 2021

Published: 9 September 2021

Publisher's Note: MDPI stays neutral with regard to jurisdictional claims in published maps and institutional affiliations.

Copyright: (c) 2021 by the authors. Licensee MDPI, Basel, Switzerland. This article is an open access article distributed under the terms and conditions of the Creative Commons Attribution (CC BY) license (https:// creativecommons.org/licenses/by/ $4.0 /)$.

\begin{abstract}
In this article, we derive representation formulas for a class of $r$-associated Stirling numbers of the second kind and examine their connections with a class of generalized Bernoulli polynomials. Herein, we use the Blissard umbral approach and the familiar Bell polynomials. Links with available literature on this subject are also pointed out. The extension to the bivariate case is discussed.
\end{abstract}

Keywords: $r$-associated Stirling numbers; representation formulas; Bernoulli polynomials; Blissard umbral approach; Bell polynomials; 2D extensions

MSC: Primary 11B73; Secondary 33C45

\section{Introduction}

Special functions and polynomials appear in the search for the explicit solutions of the most important problems in mathematical physics, electrodynamics, classical and modern physics, classical and quantum mechanics, and even in statistics and biological sciences. Among them, an important role is played by the hypergeometric functions which constitute an important class that unifies, through the introduction of appropriate parameters, most (if not all) parts of special functions, including elliptic integrals, Beta functions, incomplete Gamma functions, Bessel functions, Legendre functions, classical orthogonal polynomials, Kummer confluent functions, etc. (see, for example [1-3]).

The extension of the Pochhammer symbol allowed the introduction of many multivariate generalizations of hypergeometric functions (see [4-8]). Connections with several extensions of the celebrated Zeta function and the relevant applications to number theory and statistics have recently been highlighted in [9].

Recently, Srivastava et al. [10] introduced and studied a new kind of parametric generalization of each of the Apostol-Bernoulli polynomials of order $\alpha$, the ApostolEuler polynomials of order $\alpha$ and the Apostol-Genocchi polynomials of order $\alpha$ (see, for details [11-14] and the references therein). In fact, as an interesting application, they used such generalized parametric polynomials to explicitly give a computation of new Taylortype series containing the corresponding Apostol-Bernoulli, Euler and Genocchi numbers of order $\alpha$.

Special polynomials have a close connection with number theory, and one of the most important sets of special numbers is the class of Stirling numbers (of the first and second kind), introduced in 1730 by the Scottish mathematician James Stirling $(1692,1770)$.

Stirling numbers of the second kind are generally denoted by the symbol $\left\{\begin{array}{l}n \\ k\end{array}\right\}$ or $S(n, k)$ and are frequently used in combinatorial mathematical problems. We will use the symbol $S(n, k)$, which is typographically more simple. 
Stirling numbers of the second kind $S(n, k)$ denote the number of ways in which $n$-labelled objects can be subdivided among $k$ disjoint and non-empty subsets.

Their generating function writes:

$$
\left(e^{x}-1\right)^{k}=k ! \sum_{n=k}^{\infty} S(n, k) \frac{x^{n}}{n !} .
$$

They satisfy the recursion:

$$
S(n, k)=k S(n-1, k)+S(n-1, k-1),
$$

with the initial conditions $S(n, k)=0$ if $k=0$ or $n<k$ and $S(n, k)=1$ if $n=k$.

Several extensions of the Stirling numbers have been proposed in the literature. One of them is given by the $r$-associated Stirling numbers of the second kind, reported in [15-17]. They will be denoted by $S(n, k ; r)$ and have the following combinatorial meaning:

$r$-associated Stirling numbers of the second kind $S(n, k ; r)$ denotes the number of partitions of the set $\{1,2, \ldots, n\}$ into $k$ non-empty disjoint subsets, such that the numbers $1,2, \ldots, r$ are in distinct subsets.

Their generating function writes:

$$
\left(e^{x}-\sum_{\ell=0}^{r-1} \frac{x^{n}}{n !}\right)^{k}=k ! \sum_{n=k r}^{\infty} S(n, k ; r) \frac{x^{n}}{n !}
$$

They satisfy the recursion:

$$
S(n, k ; r)=k S(n-1, k ; r)+\left(\begin{array}{c}
n-1 \\
r-1
\end{array}\right) S(n-r, k-1 ; r),
$$

with the initial conditions $S(n, k ; r)=0$ if $k=0$ or $n<k r$ and $S(n, k ; r)=1$ if $n=k r$.

When $r=1$, the usual Stirling numbers are recovered.

The Bernoulli numbers are a sequence of rational numbers which have deep connections with number theory.

They enter in the expression of the sum of $m$ th powers of the first $n$ positive integer numbers; in the Taylor expansion of the tangent and hyperbolic tangent functions; in the Euler-Maclaurin quadrature rule; in representing certain values of the Riemann zeta function, and also have connections with Fermat's last theorem.

The Bernoulli polynomials were first generalized by L. Carlitz [18], H.M. Srivastava et al. $[11,19,20]$. More recently, several extensions have been made, as can be seen in, e.g., [21-26]. See also [11,22].

The values of the Bernoulli polynomials at the origin give the Bernoulli numbers, i.e., $B_{n}:=B_{n}(0)$. The Stirling numbers of the second kind are related to them through the equation:

$$
B_{n}=\sum_{k=0}^{n}(-1)^{k} \frac{k !}{k+1} S(n, k) .
$$

It seems that the basis of the generalizations of Bernoulli polynomials (and numbers) stands in the Mittag-Leffler function:

$$
E_{1, r+1}(x)=\frac{x^{r}}{e^{x}-\sum_{\ell=0}^{r-1} \frac{x^{\ell}}{\ell !}},
$$

considered by R.P. Agarwal in [27]. 
Actually, all extensions start from the generating function of the type:

$$
\frac{t^{\alpha r} e^{x t}}{\left(e^{x}-\sum_{\ell=0}^{r-1} \frac{x^{\ell}}{\ell !}\right)^{\alpha}}=\sum_{n=0}^{\infty} B_{n}^{(\alpha)}(t) \frac{x^{n}}{n !}
$$

where $\alpha$ is a positive real number, introduced by L. Carlitz in [18]. The generalizations include the Apostol parameter $\lambda$, in order to make the result more flexible so that many polynomial families are recovered $[11,24,28]$.

Dealing with generalized Bernoulli numbers, it is suitable to put $\alpha=k$, a positive integer.

In this article, we start from the generating function of a generalization of Bernoulli polynomials, introduced in [26] and further extended by B. Kurt [23,24], in the form:

$$
G^{[r-1, k]}(x, t)=\frac{x^{k r} e^{x t}}{\left(e^{x}-\sum_{\ell=0}^{r-1} \frac{x^{\ell}}{\ell !}\right)^{k}}=\frac{x^{k r} e^{x t}}{k ! \sum_{n=k r}^{\infty} S(n, k ; r) \frac{x^{n}}{n !}}=\sum_{n=0}^{\infty} B_{n}^{[r-1, k]}(t) \frac{x^{n}}{n !},
$$

which involves the $r$-associated Stirling numbers of the second kind. This allows to represent the coefficients of such polynomials in function of the aforementioned $r$-associated numbers. To obtain this result, a general formula for the construction of the reciprocal of a power series is introduced which makes use of the Blissard problem and Bell's polynomials.

It is worth noting that Bell polynomials are related to partitions of an integer, which have well-known symmetry properties. For example, Richard P. Stanley proved [29] that the total number of units appearing in partitions of an assigned number $n$ is equal to the total number of distinct parts that are present in the partitions. In 1984, Paul Elder generalised this result to the total number of occurrences of an integer $k$ among the partitions of $n$.

Finally, we want to stress that all the aforementioned extensions enter as particular cases of the class of Appell polynomials, whose generating function is of the type:

$$
G_{A}(x, t):=A(x) e^{t x}=\sum_{n=0}^{\infty} a_{n}(t) \frac{x^{n}}{n !} .
$$

Therefore, all the theoretical techniques exploited by G. Dattoli [30], Dattoli et al. [30-33] and Y. Ben Cheikh [34] could be used in order to derive many properties (such as recursions, shift operators, differential equations) of the resulting polynomials. However, the greater complexity of the function $A(x)$ renders its construction almost useless.

Since belonging to the Appell class makes it possible in a natural way to extend these polynomials to the multidimensional case, in the final part of the article, this extension is made, limited to the bivariate case. Of course, multidimensional extensions are also possible, however, the resulting formulas are increasingly cumbersome.

\section{Basic Definitions}

The falling factorial is given by

$$
\langle x\rangle_{n}= \begin{cases}x(x-1) \cdots(x-n+1), & n \geq 1, \\ 1, & n=0 .\end{cases}
$$

The Stirling numbers of the second kind are defined by

$$
S(n, k)=\frac{1}{k !} \sum_{m=0}^{k}(-1)^{k-m}\left(\begin{array}{c}
k \\
m
\end{array}\right) m^{n}
$$


The $r$-associate Stirling numbers of the second kind $S(n, k ; r)$ are defined by

$$
\left(\sum_{\ell=r}^{\infty} \frac{x^{\ell}}{\ell !}\right)^{k}=\left(e^{x}-\sum_{\ell=0}^{r-1} \frac{x^{\ell}}{\ell !}\right)^{k}=k ! \sum_{n=r k}^{\infty} S(n, k ; r) \frac{x^{n}}{n !} .
$$

Of course, $S(n, k ; 1)=S(n, k)$.

In the particular case when $r=2$, it results: $S(0,0 ; 2)=1, S(n, 0 ; 2)=0$, for $n \geq 1$, and:

$$
S(n, k ; 2)= \begin{cases}\frac{1}{k !} \sum_{j=0}^{k}(-1)^{j}\left(\begin{array}{l}
k \\
j
\end{array}\right) \sum_{m=0}^{j}\left(\begin{array}{c}
j \\
m
\end{array}\right)\langle n\rangle_{m}(k-j)^{n-m}, & n \geq 2 k \geq 2 ， \\
0, & 0 \leq n<2 k .\end{cases}
$$

\section{The Case When $k=1$}

Considering the case when $k=1$, we have:

$$
\sum_{\ell=r}^{\infty} \frac{x^{\ell}}{\ell !}=e^{x}-\sum_{\ell=0}^{r-1} \frac{x^{\ell}}{\ell !}=\sum_{n=r}^{\infty} S(n, 1 ; r) \frac{x^{n}}{n !}
$$

so that:

$$
\begin{gathered}
\left(\sum_{\ell=r}^{\infty} \frac{x^{\ell}}{\ell !}\right)^{2}=x^{2 r} \sum_{n=0}^{\infty} \sum_{j=0}^{n} \frac{n !}{(n-j+r) !(j+r) !} \times \\
\quad \times S(n-j+r, 1 ; r) S(j+r, 1 ; r) \frac{x^{n}}{n !}
\end{gathered}
$$

and in general:

$$
\begin{gathered}
\left(\sum_{\ell=r}^{\infty} \frac{x^{\ell}}{\ell !}\right)^{k}=x^{k r} \sum_{n=0}^{\infty} \sum_{j_{1}+j_{2}+\cdots+j_{k}=0}^{n} \frac{n !}{\left.\left(j_{1}+r\right) !\left(j_{2}+r\right) ! \cdots\left(j_{k}+r\right) !\right)} \times \\
\times S\left(j_{1}+r, 1 ; r\right) S\left(j_{2}+r, 1 ; r\right) \cdots S\left(j_{k}+r, 1 ; r\right) \frac{x^{n}}{n !} .
\end{gathered}
$$

Writing Equation (3) in the form

$$
\begin{gathered}
\left(\sum_{\ell=r}^{\infty} \frac{x^{\ell}}{\ell !}\right)^{k}=k ! \sum_{n=0}^{\infty} S(n+k r, k ; r) \frac{x^{n+k r}}{(n+k r) !}= \\
=k ! x^{k r} \sum_{n=0}^{\infty} \frac{n !}{(n+k r) !} S(n+k r, k ; r) \frac{x^{n}}{n !}
\end{gathered}
$$

and comparing this equation with (7), we find the result:

Theorem 1. For any fixed integers $k$ and $r$, the $r$-associate Stirling numbers of the second kind $S(n+k r, k ; r)$ are connected with that relative to lower element numbers and $k=1$, by means of the equation:

$$
\begin{gathered}
\frac{k !}{(n+k r) !} S(n+k r, k ; r)= \\
=\sum_{j_{1}+j_{2}+\cdots+j_{k}=0}^{n} \frac{1}{\left(j_{1}+r\right) !\left(j_{2}+r\right) ! \cdots\left(j_{k}+r\right) !} \times \\
\times S\left(j_{1}+r, 1 ; r\right) S\left(j_{2}+r, 1 ; r\right) \cdots S\left(j_{k}+r, 1 ; r\right),
\end{gathered}
$$




\section{The Blissard Problem}

According to the Blissard problem [17], the reciprocal of a Taylor series can be expressed in terms of Bell polynomials. In fact, consider the sequences $a:=\left\{a_{k}\right\}=$ $\left(1, a_{1}, a_{2}, a_{3}, \ldots\right)$, and $b:=\left\{b_{k}\right\}=\left(b_{0}, b_{1}, b_{2}, b_{3}, \ldots\right)$, and the function:

$$
\frac{1}{1+a_{1} t+a_{2} \frac{t^{2}}{2 !}+a_{3} \frac{t^{3}}{3 !}+\ldots} \quad(t \geq 0) .
$$

Using the umbral formalism (that is, letting $a_{k} \equiv a^{k}$ and $b_{k} \equiv b^{k}$ ), the solution of the equation:

$$
\frac{1}{\sum_{n=0}^{\infty} \frac{a^{n} t^{n}}{n !}}=\sum_{n=0}^{\infty} \frac{b^{n} t^{n}}{n !}, \quad \text { i.e. } \quad \exp [a t] \exp [b t]=1
$$

is given by

$$
\left\{\begin{array}{l}
b_{0}:=1, \\
b_{n}=Y_{n}\left(-1 !, a_{1} ; 2 !, a_{2} ;-3 !, a_{3} ; \ldots ;(-1)^{n} n !, a_{n}\right), \quad(\forall n>0),
\end{array}\right.
$$

where $Y_{n}$ is the $n$th Bell polynomial [17].

The Bell polynomials satisfy the equation

$$
Y_{n}\left(f_{1}, \beta g_{1} ; f_{2}, \beta^{2} g_{2} ; \ldots ; f_{n}, \beta^{n} g_{n}\right)=\beta^{n} Y_{n}\left(f_{1}, g_{1} ; f_{2}, g_{2} ; \ldots ; f_{n}, g_{n}\right) .
$$

Furthermore, they are usually written in the form:

$$
Y_{n}\left(f_{1}, g_{1} ; f_{2}, g_{2} ; \ldots ; f_{n}, g_{n}\right)=\sum_{h=1}^{n} B_{n, h}\left(g_{1}, g_{2}, \ldots, g_{n-h+1}\right) f_{h},
$$

where $B_{n, h}$ are called Bell polynomials of the second kind and satisfy the recursion [35]:

$$
B_{n, h}\left(g_{1}, g_{2}, \ldots, g_{n-h+1}\right)=\sum_{\ell=0}^{n-h}\left(\begin{array}{c}
n-1 \\
\ell
\end{array}\right) B_{n-\ell-1, h-1}\left(g_{1}, g_{2}, \ldots, g_{n-\ell-h+1}\right) g_{\ell+1} .
$$

For any $h=1,2, \ldots, n$, the $B_{n, h}$ polynomials satisfy the equation:

$$
B_{n, h}\left(\alpha \beta g_{1}, \alpha \beta^{2} g_{2}, \ldots, \alpha \beta^{n-h+1} g_{n-h+1}\right)=\alpha^{h} \beta^{n} B_{n, h}\left(g_{1}, g_{2}, \ldots, g_{n-h+1}\right) .
$$

This means that they are polynomials of the $g_{1}, g_{2}, \ldots, g_{n}$ variables, homogeneous of degree $h$ and isobaric of weight $n$ (i.e., they are linear combinations of monomials $g_{1}^{h_{1}} g_{2}^{h_{2}} \cdots g_{n}^{h_{n}}$ whose weight is constantly given by $\left.h_{1}+2 h_{2}+\ldots+n h_{n}=n\right)$.

By using Equation (14), the Function (10) writes:

$$
\frac{1}{\sum_{n=0}^{\infty} \frac{a_{n} t^{n}}{n !}}=1+\sum_{n=1}^{\infty} \sum_{h=1}^{n}(-1)^{h} h ! B_{n, h}\left(a_{1}, a_{2}, \ldots, a_{n-h+1}\right) \frac{t^{n}}{n !} .
$$

It is convenient to introduce the definition:

$$
C_{n}(a):=\sum_{h=1}^{n}(-1)^{h} h ! B_{n, h}\left(a_{1}, a_{2}, \ldots, a_{n-h+1}\right), \quad C_{0}(a):=1,
$$


so that Equation (17) becomes:

$$
\frac{1}{\sum_{n=0}^{\infty} \frac{a_{n} t^{n}}{n !}}=\sum_{n=0}^{\infty} C_{n}(a) \frac{t^{n}}{n !}
$$

\section{A Simple Application}

The Blissard problem can be used in solving the following.

Problem 1. Given the power series $A(t):=\sum_{n=0}^{\infty} a_{n} t^{n} / n !$ and $B(t):=\sum_{n=0}^{\infty} b_{n} t^{n} / n !$, where $a_{0}=b_{0}=1$, find the coefficients $z_{n}$ of the series representing the ratio:

$$
\frac{A(t)}{B(t)}=\sum_{n=0}^{\infty} z_{n} \frac{t^{n}}{n !}
$$

According to Equation (19), introducing the umbral symbol $b=\left(1, b_{1}, b_{2} \ldots\right)$, we can write:

$$
\frac{A(t)}{B(t)}=\sum_{n=0}^{\infty} a_{n} \frac{t^{n}}{n !} \sum_{n=0}^{\infty} C_{n}(b) \frac{t^{n}}{n !}=\sum_{n=0}^{\infty} \sum_{h=0}^{n}\left(\begin{array}{l}
n \\
h
\end{array}\right) a_{n-h} C_{h}(b) \frac{t^{n}}{n !}=\sum_{n=0}^{\infty} z_{n} \frac{t^{n}}{n !},
$$

and therefore, we conclude that:

$$
z_{n}=\sum_{h=0}^{n}\left(\begin{array}{l}
n \\
h
\end{array}\right) a_{n-h} C_{h}(b)
$$

Remark 1. Note that the link between the coefficients of the above power series could even be derived from the equation:

$$
\sum_{n=0}^{\infty} a_{n} \frac{t^{n}}{n !}=\sum_{n=0}^{\infty} b_{n} \frac{t^{n}}{n !} \sum_{n=0}^{\infty} z_{n} \frac{t^{n}}{n !}=\sum_{n=0}^{\infty} \sum_{h=0}^{n}\left(\begin{array}{l}
n \\
h
\end{array}\right) b_{n-h} z_{h} \frac{t^{n}}{n !}
$$

so that:

$$
a_{n}=\sum_{h=0}^{n}\left(\begin{array}{l}
n \\
h
\end{array}\right) b_{n-h} z_{h}
$$

but this method needs to find the $z_{n}$ solving a system.

For example, we find:

$$
\begin{aligned}
& a_{0}=z_{0} b_{0}=1 \\
& a_{1}=b_{1} z_{0}+b_{0} z_{1} \\
& a_{2}=b_{2} z_{0}+2 b_{1} z_{1}+b_{0} z_{2} \\
& \cdots \\
& a_{n}=(b+z)^{n}, \text { with } b^{h} \equiv b_{h}, z^{h} \equiv z_{h}
\end{aligned}
$$

so that:

$$
\begin{aligned}
& z_{0}=a_{0}=1 \\
& z_{1}=a_{1}-b_{1} \\
& z_{2}=a_{2}-b_{2}-2 b_{1}\left(a_{1}-b_{1}\right) \\
& z_{3}=a_{3}-b_{3}-3 b_{2}\left(a_{1}-b_{1}\right)-3 b_{1}\left[a_{2}-b_{2}-2 b_{1}\left(a_{1}-b_{1}\right)\right] \\
& \ldots
\end{aligned}
$$

Although the system lends itself to a recurring calculation, it provides the coefficients $z_{n}$ by means of a system of Chinese boxes and does not provide an explicit formula such as that obtained 
using Bell's polynomials. Of course, recursion remains hidden in Bell's polynomial computation, which nevertheless seems to be simpler than that required for the solution of the system (25).

In the Appendix $\mathrm{A}$ at the end of this article, the first few values of the solution $z_{n}$ $(n=1,2, \ldots, 7)$ were computed by the third author by using Mathematica ${ }^{\odot}$. The results can be extended to higher values of $n$, but the polynomial expression in terms of the coefficients $a$ and $b$ become more and more cumbersome.

It is worth noting that the sum of indexes of the $a$ and $b$ coefficients in the representation of $z_{n}$ is given by $n, \forall n \geq 1$.

Remark 2. Note that in the above considered problem, we assumed a normalization of the considered series assuming $a_{0}=b_{0}=1$. If this condition is not satisfied, but we still have the condition $a_{0} \neq 0, b_{0} \neq 0$, we can use the same method, putting $\alpha:=\left\{\alpha_{n}\right\}=\left(1, a_{1} / a_{0}, a_{2} / a_{0}, \ldots\right)$, $\beta:=\left\{\beta_{n}\right\}=\left(1, b_{1} / b_{0}, b_{2} / b_{0}, \ldots\right)$, and considering the problem in the form:

$$
\frac{a_{0}}{b_{0}}\left(\frac{\sum_{n=0}^{\infty} \alpha_{n} \frac{t^{n}}{n !}}{\sum_{n=0}^{\infty} \beta_{n} \frac{t^{n}}{n !}}\right)=\sum_{n=0}^{\infty} z_{n} \frac{t^{n}}{n !} .
$$

Denoting by $\zeta_{n}$ the coefficients of the series representing the ratio in brackets in Equation (27), the solution of the problem (27) will be given by $z_{n}=\frac{a_{0}}{b_{0}} \zeta_{n}$.

\section{Generalized Bernoulli Polynomials}

In [26], a generalization of the Bernoulli polynomials and numbers was introduced, by means of the generating function:

$$
G^{[r-1]}(x, t)=\frac{x^{r} e^{x t}}{e^{x}-\sum_{\ell=0}^{r-1} \frac{x^{\ell}}{\ell !}}=\sum_{n=0}^{\infty} B_{n}^{[r-1]}(t) \frac{x^{n}}{n !} .
$$

Obviously, this results in $B_{n}^{[0]}(t) \equiv B_{n}(t)$, the classical Bernoulli polynomials.

According to Equation (5), it results that:

$$
\frac{x^{r} e^{x t}}{\sum_{n=r}^{\infty} S(n, 1 ; r) \frac{x^{n}}{n !}}=\sum_{n=0}^{\infty} B_{n}^{[r-1]}(t) \frac{x^{n}}{n !},
$$

or, in equivalent form:

$$
\frac{\sum_{n=0}^{\infty} t^{n} \frac{x^{n}}{n !}}{\sum_{n=0}^{\infty} \frac{n !}{(n+r) !} S(n+r, 1 ; r) \frac{x^{n}}{n !}}=\sum_{n=0}^{\infty} B_{n}^{[r-1]}(t) \frac{x^{n}}{n !},
$$

Of course, from Equation (28), we have:

$$
S(n, 1 ; r)=1, \quad \forall n \geq r .
$$

\section{A Larger Class of Bernoulli Polynomials}

A natural extension of this class of polynomials was obtained by B. Kurt [23,24], considering, for any fixed integer $k$, the generating function: 


$$
G^{[r-1, k]}(x, t)=\frac{x^{k r} e^{x t}}{\left(e^{x}-\sum_{\ell=0}^{r-1} \frac{x^{\ell}}{\ell !}\right)^{k}}=\frac{x^{k r} e^{x t}}{k ! \sum_{n=k r}^{\infty} S(n, k ; r) \frac{x^{n}}{n !}}=\sum_{n=0}^{\infty} B_{n}^{[r-1, k]}(t) \frac{x^{n}}{n !},
$$

so that $B_{n}^{[0,1]}(t) \equiv B_{n}(t)$. By noting that:

$$
\sum_{n=k r}^{\infty} S(n, k ; r) \frac{x^{n}}{n !}=x^{k r} \sum_{n=0}^{\infty} \frac{n !}{(n+k r) !} S(n+k r, k ; r) \frac{x^{n}}{n !},
$$

the above-generating function writes:

$$
G^{[r-1, k]}(x, t)=\frac{\sum_{n=0}^{\infty} t^{n} \frac{x^{n}}{n !}}{k ! \sum_{n=0}^{\infty} \frac{n !}{(n+k r) !} S(n+k r, k ; r) \frac{x^{n}}{n !}}=\sum_{n=0}^{\infty} B_{n}^{[r-1, k]}(t) \frac{x^{n}}{n !},
$$

\section{Representation Formulas}

A direct application of the problem in Section 4 gives representation formulas for the generalized Bernoulli polynomials in Equations (30) and (33) in terms of $r$-associate Stirling numbers of the second kind, expressed by the following theorems.

Theorem 2. The generalized Bernoulli polynomials, defined in Equation (33), can be represented in terms of the r-associate Stirling numbers of the second kind (of the type $S(n, k ; r)$ ), by means of the equation:

$$
B_{n}^{[r-1, k]}(t)=\sum_{h=0}^{n}\left(\begin{array}{l}
n \\
h
\end{array}\right) C_{h}(\beta) t^{n-h}
$$

where $\beta=\left(1, \beta_{1}, \beta_{2}, \ldots\right)$, with $\beta_{n}=\frac{k ! n !}{(n+k r) !} S(n+k r, k ; r)$, and the symbol $C_{h}(\cdot)$ is defined in Equation (18).

Proof. It is sufficient to apply Equation (27), assuming:

$$
\begin{aligned}
& \alpha_{n}=t^{n} \\
& \beta_{n}=\frac{n !(k r) !}{(n+k r) !} \frac{S(n+k r, k ; r)}{S(k r, k ; r)} \\
& z_{n}=B_{n}^{[r-1, k]}(t) .
\end{aligned}
$$

\section{The Generalized Bernoulli Numbers}

As a by-product of the preceding results, we find the relations relevant to the generalized Bernoulli numbers $B_{n}^{[r-1, k]}:=B_{n}^{[r-1, k]}(0)$.

The generating function of the generalized Bernoulli numbers is given by

$$
G^{[r-1, k]}(x)=\frac{x^{k r}}{\left(e^{x}-\sum_{\ell=0}^{r-1} \frac{x^{\ell}}{\ell !}\right)^{k}}=\sum_{n=0}^{\infty} B_{n}^{[r-1, k]} \frac{x^{n}}{n !},
$$


or, in equivalent form, involving the $S(n+k r, k ; r)$ numbers:

$$
G^{[r-1, k]}(x)=\frac{1}{k ! \sum_{n=0}^{\infty} \frac{n !}{(n+k r) !} S(n+k r, k ; r) \frac{x^{n}}{n !}}=\sum_{n=0}^{\infty} B_{n}^{[r-1, k]} \frac{x^{n}}{n !},
$$

By exploiting one of the techniques for finding the reciprocal of a Taylor series described in Section 4, from the knowledge of the generalized Bernoulli numbers, the $r$-associate Stirling numbers of the second kind can be derived, so that a useful check with the known tables of the $r$-associate Stirling numbers of the second kind can be obtained.

In Figures 1-4, these numbers are reported for the values $k=1,2,3,4, r=2,3,4,5$ and $n=1,2, \ldots, 10$.

Further results can be obtained by using the computer algebra program Mathematica ${ }^{\odot}$.

$r=2$

$B_{n}^{[r-1, k]}$

\begin{tabular}{|c|c|c|c|c|}
\hline & $k=1$ & $k=2$ & $k=3$ & $k=4$ \\
\hline$n=0$ & 2 & 4 & 8 & 16 \\
\hline$n=1$ & $-2 / 3$ & $-8 / 3$ & -8 & $-64 / 3$ \\
\hline$n=2$ & $1 / 9$ & $4 / 3$ & $20 / 3$ & $224 / 9$ \\
\hline$n=3$ & $1 / 45$ & $-16 / 45$ & $-188 / 45$ & $-1088 / 45$ \\
\hline$n=4$ & $-1 / 135$ & $-2 / 27$ & $64 / 45$ & $2684 / 135$ \\
\hline$n=5$ & $-5 / 567$ & $4 / 63$ & $64 / 189$ & $-3968 / 567$ \\
\hline$n=6$ & $-1 / 2835$ & $22 / 405$ & $-1234 / 2835$ & $-128 / 63$ \\
\hline$n=7$ & $7 / 1215$ & $-32 / 1215$ & $-46 / 135$ & $256 / 81$ \\
\hline$n=8$ & $13 / 3645$ & $-82 / 1215$ & $28 / 81$ & $3032 / 1215$ \\
\hline$n=9$ & $-307 / 66825$ & $4 / 66825$ & $8116 / 13365$ & $-49696 / 13365$ \\
\hline$n=10$ & $-479 / 56133$ & $2914 / 25515$ & $-6422 / 18711$ & $-315184 / 56133$ \\
\hline
\end{tabular}

Figure 1. Numbers $B_{n}^{[1, k]} ; k=1,2,3,4 ; n=0,1, \ldots, 10$.

$$
\begin{aligned}
& r=3 \\
& B_{n}^{[r-1, k]}
\end{aligned}
$$

\begin{tabular}{|c|c|c|c|c|}
\hline & $k=1$ & $k=2$ & $k=3$ & $k=4$ \\
\hline$n=0$ & 6 & 36 & 216 & 1296 \\
\hline$n=1$ & $-3 / 2$ & -18 & -162 & -1296 \\
\hline$n=2$ & $3 / 20$ & $63 / 10$ & $486 / 5$ & $5508 / 5$ \\
\hline$n=3$ & $3 / 80$ & $-9 / 10$ & $-81 / 2$ & $-3726 / 5$ \\
\hline$n=4$ & $3 / 2800$ & $-423 / 1400$ & $4617 / 700$ & $24219 / 70$ \\
\hline$n=5$ & $-3 / 448$ & $9 / 560$ & $243 / 80$ & $-405 / 7$ \\
\hline$n=6$ & $-13 / 3200$ & $1173 / 11200$ & $-27 / 140$ & $-5157 / 140$ \\
\hline$n=7$ & $7 / 12800$ & $21 / 400$ & $-243 / 160$ & $27 / 40$ \\
\hline$n=8$ & $7453 / 2464000$ & $-9609 / 246400$ & $-93879 / 123200$ & $1465587 / 61600$ \\
\hline$n=9$ & $20007 / 9856000$ & $-7767 / 98560$ & $42039 / 44800$ & $844911 / 61600$ \\
\hline$n=10$ & $-344259 / 256256000$ & $-2792007 / 128128000$ & $53277507 / 32032000$ & $-318338019 / 16016000$ \\
\hline
\end{tabular}

Figure 2. Numbers $B_{n}^{[2, k]} ; k=1,2,3,4 ; n=0,1, \ldots, 10$. 


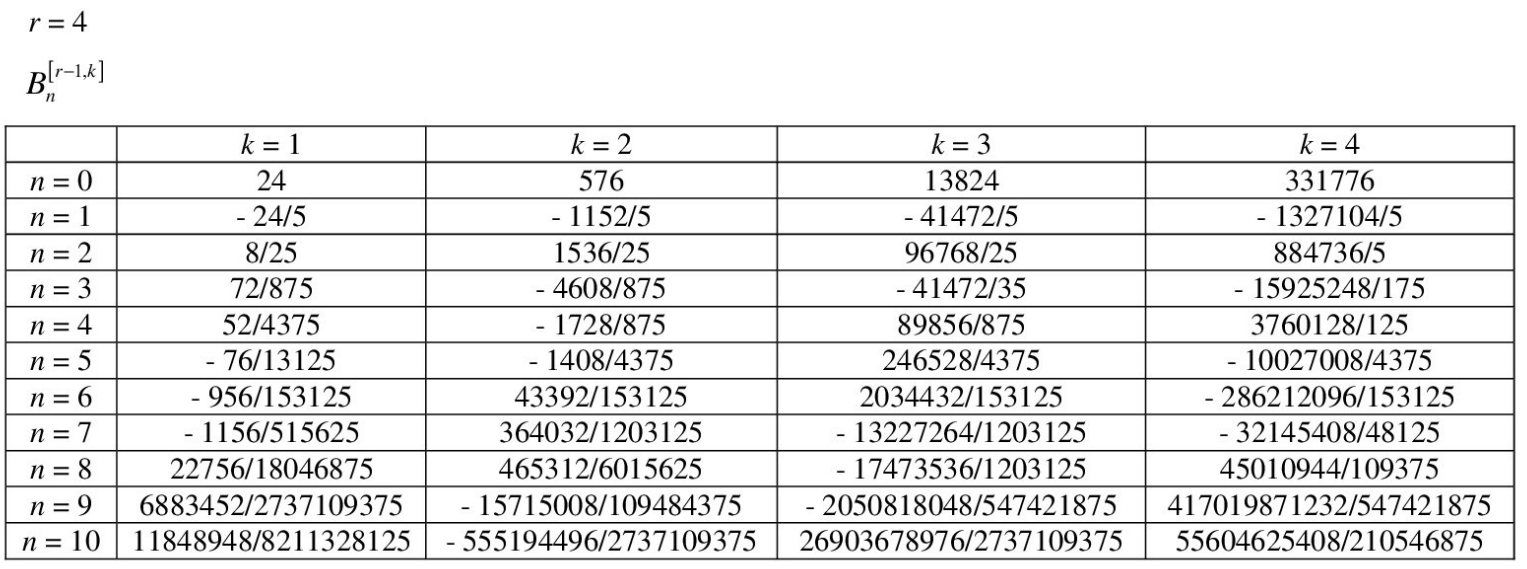

Figure 3. Numbers $B_{n}^{[3, k]} ; k=1,2,3,4 ; n=0,1, \ldots, 10$.

$\begin{aligned} & r=5 \\
& B_{n}^{[r-1, k]}\end{aligned}$
\begin{tabular}{|c|c|c|c|c|}
\hline & $k=1$ & $k=2$ & $k=3$ & $k=4$ \\
\hline$n=0$ & 120 & 14400 & 1728000 & 207360000 \\
\hline$n=1$ & -20 & -4800 & -864000 & -138240000 \\
\hline$n=2$ & $20 / 21$ & $7200 / 7$ & $2304000 / 7$ & $-218880000 / 7$ \\
\hline$n=3$ & $5 / 21$ & $-400 / 7$ & $-552000 / 7$ & $387840000 / 49$ \\
\hline$n=4$ & $20 / 441$ & $-3200 / 147$ & $192000 / 49$ & $-14080000 / 49$ \\
\hline$n=5$ & $-5 / 1323$ & $-800 / 147$ & $16000 / 7$ & $-1018560000 / 3773$ \\
\hline$n=6$ & $-2155 / 203742$ & $27100 / 33957$ & $9304000 / 11319$ & $-76480000 / 539$ \\
\hline$n=7$ & $-155 / 24948$ & $30200 / 14553$ & $-32000 / 539$ & $-64640000 / 7007$ \\
\hline$n=8$ & $-10480 / 11918907$ & $1663600 / 1324323$ & $-18064000 / 49049$ & $1398080000 / 21021$ \\
\hline$n=9$ & $36215 / 15891876$ & $-38200 / 1324323$ & $-119932000 / 441441$ & $28905920000 / 441441$ \\
\hline$n=10$ & $230105 / 83432349$ & $-2219500 / 2528253$ & $3056000 / 441441$ & \\
\hline
\end{tabular}

Figure 4. Numbers $B_{n}^{[4, k]} ; k=1,2,3,4 ; n=0,1, \ldots, 10$.

\section{2D Extensions of the Bernoulli and Appell Polynomials}

In a preceding article [33], the Hermite-Kampé de Fériet [36] (or Gould-Hopper) polynomials [37,38], were used in order to extend the multivariate case to several polynomial sets.

By changing the notation, in order to preserve that used in the considered literature, these polynomials are defined by the generating function:

$$
e^{t x+\tau x^{j}}=\sum_{n=0}^{\infty} H_{n}^{(j)}(t, \tau) \frac{x^{n}}{n !}
$$

or by the explicit form:

$$
H_{n}^{(j)}(t, \tau)=n ! \sum_{s=0}^{\left[\frac{n}{j}\right]} \frac{t^{n-j s} \tau^{s}}{(n-j s) ! s !},
$$

where $j \geq 2$ is a positive integer.

When $j=2$ bivariate extensions of the Bernoulli and Euler polynomials were introduced [39], the case of higher dimensions was also considered in [32].

As recalled in Section 5, generalized Bernoulli polynomials are a special case of Appell polynomials, and the 2D extension of Appell polynomials introduced in [21] can be used for introducing the 2D generalized Bernoulli polynomials. 


\section{D Appell Polynomials}

We recall that, for any $j \geq 2$, the bivariate Appell polynomials $R_{n}^{(j)}(t, \tau)$ are defined in [21], by means of the generating function:

$$
G_{A}^{(j)}(t, \tau ; x):=A(x) e^{t x+\tau x^{j}}=\sum_{n=0}^{\infty} R_{n}^{(j)}(t, \tau) \frac{x^{n}}{n !}
$$

Assuming $A_{[r, k]}(x):=\frac{x^{k r}}{\left(e^{x}-\sum_{\ell=0}^{r-1} \frac{x^{\ell}}{\ell !}\right)^{k}}$, we have the definition of bivariate generalized Bernoulli polynomials through the generating function:

$$
G_{A_{[r, k]}^{(j)}}(t, \tau ; x):=\frac{x^{k r}}{\left(e^{x}-\sum_{\ell=0}^{r-1} \frac{x^{\ell}}{\ell !}\right)^{k}} e^{t x+\tau x^{j}}=\sum_{n=0}^{\infty} R_{n}^{(j ; r, k)}(t, \tau) \frac{x^{n}}{n !}
$$

In [21], we have derived explicit forms of the polynomials $R_{n}^{(j)}(t, \tau)$ in terms of the Hermite-Kampé de Fériet polynomials $H_{n}^{(j)}$ and vice versa.

Therefore, in the case of the considered $R_{n}^{(j ; r, k)}(t, \tau)$ polynomials, we find the result.

Theorem 3. The explicit form of the $R_{n}^{(j ; r, k)}(t, \tau)$ polynomials is expressed, in terms of the HermiteKampé de Fériet polynomials, by

$$
R_{n}^{(j ; r, k)}(t, \tau)=\sum_{h=0}^{n}\left(\begin{array}{l}
n \\
h
\end{array}\right) \mathcal{R}_{n-h}^{[r, k]} H_{h}^{(j)}(t, \tau) n ! \sum_{h=0}^{n} \frac{\mathcal{R}_{n-h}^{[r, k]}}{(n-h) !} \sum_{r=0}^{\left[\frac{h}{j}\right]} \frac{x^{h-j r} y^{r}}{(h-j r) ! r !},
$$

where the $\mathcal{R}_{\ell}^{[r, k]}$ are the "Appell numbers" appearing in the definition: $A_{[r, k]}(x)=\sum_{\ell=0}^{\infty} \frac{\mathcal{R}_{\ell}^{[r, k]}}{\ell !} x^{\ell}$. Since $\left(A_{[r, k]}(0) \neq 0\right)$, the above equation can be reversed, obtaining:

$$
H_{n}^{(j)}(t, \tau)=\sum_{\ell=0}^{n}\left(\begin{array}{l}
n \\
\ell
\end{array}\right) Q_{n-\ell}^{[r, k]} R_{\ell}^{(j ; r, k)}(t, \tau),
$$

where the $Q_{\ell}^{[r, k]}$ are the coefficients of the Taylor expansion in a neighbourhood of the origin of the reciprocal function $1 / A_{[r, k]}(x)$, which can be obtained by using the results of Section 4.

Remark 3. Note that the first few values of the "Appell numbers" $\mathcal{R}_{\ell}^{[r, k]}$ in Equation (39) are just the generalized Bernoulli numbers, reported in tables appearing in Figures 1-4, for $r=2,3,4,5$, $k=1,2,3,4$ and $n=1,2, \ldots, 10$, while the coefficients $Q_{\ell}^{[r, k]}$ of the function $1 / A_{[r, k]}(x)$ in Equation (40) are given by $\frac{k ! n !}{(n+k r) !} S(n+k r, k ; r)$ whose first few values $S(n+k r, k ; r)$ can be found in Figures 5-8, for $r=2,3,4,5, k=1,2,3,4$ and $n=1,2, \ldots, 10$.

In the following Figures 5-8, we report the numerical values of the $r$-associate Stirling numbers of the second kind $S(n+r k, k ; r)$, which enter the generating function of Equation (36). 
$r=2$

$S(n+k r, k ; r)$

\begin{tabular}{|r|r|r|r|r|}
\hline & \multicolumn{1}{|c|}{$k=1$} & \multicolumn{1}{c|}{$k=2$} & \multicolumn{1}{c|}{$k=3$} & \multicolumn{1}{c|}{$k=4$} \\
\hline$n=0$ & $S(2,1 ; 2)=1$ & $S(4,2 ; 2)=3$ & $S(6,3 ; 2)=15$ & $S(8,4 ; 2)=105$ \\
\hline$n=1$ & $S(3,1 ; 2)=1$ & $S(5,2 ; 2)=10$ & $S(7,3 ; 2)=105$ & $S(9,4 ; 2)=1260$ \\
\hline$n=2$ & $S(4,1 ; 2)=1$ & $S(6,2 ; 2)=25$ & $S(8,3 ; 2)=490$ & $S(10,4 ; 2)=9450$ \\
\hline$n=3$ & $S(5,1 ; 2)=1$ & $S(7,2 ; 2)=56$ & $S(9,3 ; 2)=1918$ & $S(11,4 ; 2)=56980$ \\
\hline$n=4$ & $S(6,1 ; 2)=1$ & $S(8,2 ; 2)=119$ & $S(10,3 ; 2)=6825$ & $S(12,4 ; 2)=302995$ \\
\hline$n=5$ & $S(7,1 ; 2)=1$ & $S(9,2 ; 2)=246$ & $S(11,3 ; 2)=22935$ & $S(13,4 ; 2)=1487200$ \\
\hline$n=6$ & $S(8,1 ; 2)=1$ & $S(10,2 ; 2)=501$ & $S(12,3 ; 2)=74316$ & $S(14,4 ; 2)=6914908$ \\
\hline$n=7$ & $S(9,1 ; 2)=1$ & $S(11,2 ; 2)=1012$ & $S(13,3 ; 2)=235092$ & $S(15,4 ; 2)=30950920$ \\
\hline$n=8$ & $S(10,1 ; 2)=1$ & $S(12,2 ; 2)=2035$ & $S(14,3 ; 2)=731731$ & $S(16,4 ; 2)=134779645$ \\
\hline$n=9$ & $S(11,1 ; 2)=1$ & $S(13,2 ; 2)=4082$ & $S(15,3 ; 2)=2252341$ & $S(17,4 ; 2)=575156036$ \\
\hline$n=10$ & $S(12,1 ; 2)=1$ & $S(14,2 ; 2)=8177$ & $S(16,3 ; 2)=6879678$ & $S(18,4 ; 2)=2417578670$ \\
\hline
\end{tabular}

Figure 5. Numbers $S(n+2 k, k ; 2), k=1,2,3,4 ; n=1,2, \ldots, 10$.

$$
r=3
$$

$S(n+k r, k ; r)$

\begin{tabular}{|r|r|r|r|r|}
\hline & \multicolumn{1}{|c|}{$k=1$} & \multicolumn{1}{c|}{$k=2$} & \multicolumn{1}{c|}{$k=3$} & \multicolumn{1}{c|}{$k=4$} \\
\hline$n=0$ & $S(3,1 ; 3)=1$ & $S(6,2 ; 3)=10$ & $S(9,3 ; 3)=280$ & $S(12,4 ; 3)=15400$ \\
\hline$n=1$ & $S(4,1 ; 3)=1$ & $S(7,2 ; 3)=35$ & $S(10,3 ; 3)=2100$ & $S(13,4 ; 3)=200200$ \\
\hline$n=2$ & $S(5,1 ; 3)=1$ & $S(8,2 ; 3)=91$ & $S(11,3 ; 3)=10395$ & $S(14,4 ; 3)=1611610$ \\
\hline$n=3$ & $S(6,1 ; 3)=1$ & $S(9,2 ; 3)=210$ & $S(12,3 ; 3)=42735$ & $S(15,4 ; 3)=10335325$ \\
\hline$n=4$ & $S(7,1 ; 3)=1$ & $S(10,2 ; 3)=456$ & $S(13,3 ; 3)=158301$ & $S(16,4 ; 3)=57962905$ \\
\hline$n=5$ & $S(8,1 ; 3)=1$ & $S(11,2 ; 3)=957$ & $S(14,3 ; 3)=549549$ & $S(17,4 ; 3)=297797500$ \\
\hline$n=6$ & $S(9,1 ; 3)=1$ & $S(12,2 ; 3)=1969$ & $S(15,3 ; 3)=1827826$ & $S(18,4 ; 3)=1439774336$ \\
\hline$n=7$ & $S(10,1 ; 3)=1$ & $S(13,2 ; 3)=4004$ & $S(16,3 ; 3)=5903898$ & $S(19,4 ; 3)=6662393738$ \\
\hline$n=8$ & $S(11,1 ; 3)=1$ & $S(14,2 ; 3)=8086$ & $S(17,3 ; 3)=18682014$ & $S(20,4 ; 3)=29844199346$ \\
\hline$n=9$ & $S(12,1 ; 3)=1$ & $S(15,2 ; 3)=16263$ & $S(18,3 ; 3)=58257810$ & $S(21,4 ; 3)=130445781284$ \\
\hline$n=10$ & $S(13,1 ; 3)=1$ & $S(16,2 ; 3)=32631$ & $S(19,3 ; 3)=179765973$ & $S(22,4 ; 3)=559533979466$ \\
\hline
\end{tabular}

Figure 6. Numbers $S(n+3 k, k ; 3), k=1,2,3,4 ; n=1,2, \ldots, 10$. 


\begin{tabular}{|c|c|c|c|c|}
\hline & $k=1$ & $k=2$ & $k=3$ & $k=4$ \\
\hline$n=0$ & $S(4,1 ; 4)=1$ & $S(8,2 ; 4)=35$ & $S(12,3 ; 4)=5775$ & $S(16,4 ; 4)=2627625$ \\
\hline$n=1$ & $S(5,1 ; 4)=1$ & $S(9,2 ; 4)=126$ & $S(13,3 ; 4)=45045$ & $S(17,4 ; 4)=35735700$ \\
\hline$n=2$ & $S(6,1 ; 4)=1$ & $S(10,2 ; 4)=336$ & $S(14,3 ; 4)=231231$ & $S(18,4 ; 4)=300179880$ \\
\hline$n=3$ & $S(7,1 ; 4)=1$ & $S(11,2 ; 4)=792$ & $S(15,3 ; 4)=981981$ & $S(19,4 ; 4)=2002016016$ \\
\hline$n=4$ & $S(8,1 ; 4)=1$ & $S(12,2 ; 4)=1749$ & $S(16,3 ; 4)=3741738$ & $S(20,4 ; 4)=11633808186$ \\
\hline$n=5$ & $S(9,1 ; 4)=1$ & $S(13,2 ; 4)=3718$ & $S(17,3 ; 4)=13307294$ & $S(21,4 ; 4)=61705547904$ \\
\hline$n=6$ & $S(10,1 ; 4)=1$ & $S(14,2 ; 4)=7722$ & $S(18,3 ; 4)=45172842$ & $S(22,4 ; 4)=306902071476$ \\
\hline$n=7$ & $S(11,1 ; 4)=1$ & $S(15,2 ; 4)=15808$ & $S(19,3 ; 4)=148417854$ & $S(23,4 ; 4)=1456171781064$ \\
\hline$n=8$ & $S(12,1 ; 4)=1$ & $S(16,2 ; 4)=32071$ & $S(20,3 ; 4)=476330361$ & $S(24,4 ; 4)=6668268193587$ \\
\hline$n=9$ & $S(13,1 ; 4)=1$ & $S(17,2 ; 4)=64702$ & $S(21,3 ; 4)=1502751363$ & $S(25,4 ; 4)=29714641533060$ \\
\hline$n=10$ & $S(14,1 ; 4)=1$ & $S(18,2 ; 4)=130084$ & $S(22,3 ; 4)=4681265809$ & $S(26,4 ; 4)=129625477492940$ \\
\hline
\end{tabular}

Figure 7. Numbers $S(n+4 k, k ; 4), k=1,2,3,4 ; n=1,2, \ldots, 10$.

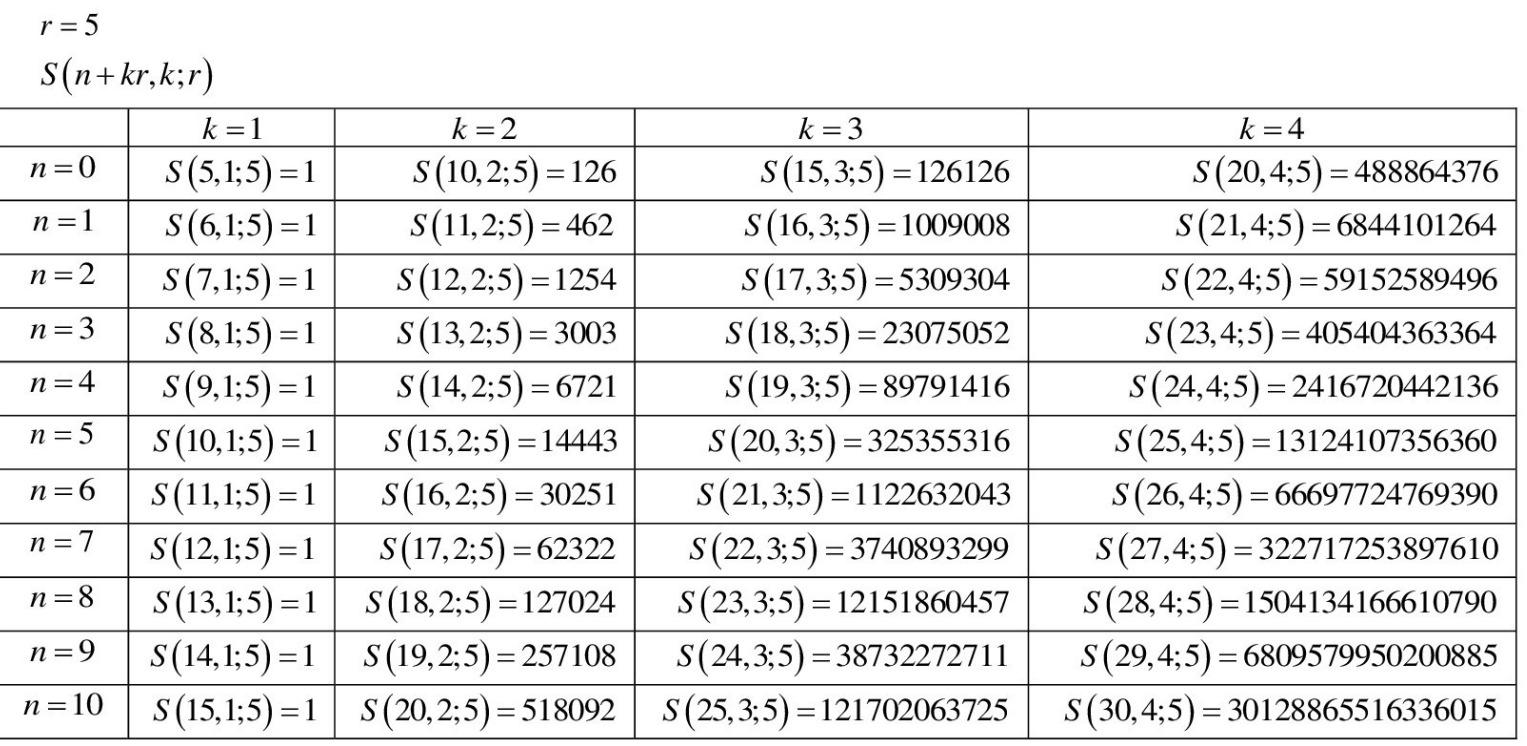

Figure 8. Numbers $S(n+5 k, k ; 5), k=1,2,3,4 ; n=1,2, \ldots, 10$.

\section{Conclusions}

Generalizations of Bernoulli polynomials have also recently received some attention due to their connections to extensions of second-kind Stirling numbers, which are of considerable interest in combinatorial mathematics. In this paper, it has been shown that such connections can be derived using a simple formula, which is useful to construct the reciprocal function of an assigned power series. This formula is based on the umbral calculus of Blissard and makes use of Bell polynomials, one of the powerful tools, born from the differential calculus $[40,41]$, whose applications range from the extension of the Newton-Girard formulas (and consequently, the reduction formulas for the orthogonal invariants of a strictly positive compact operator [42]) to combinatorics (see, e.g., [43] and the references therein).

The fact that all the generalizations considered belong to the class of Appell polynomials (the simplest among the Sheffer polynomials) also makes possible the construction of 
multidimensional versions of such polynomials, and it would be interesting to see whether multivariate versions of Stirling numbers could be constructed in an elementary way.

Author Contributions: Conceptualization, P.E.R., R.S. and P.N.; Data curation, R.S.; Investigation, P.N.; Methodology, P.E.R.; Software, P.N.; Supervision, R.S.; Validation, P.N.; Writing—original draft, P.E.R. All authors have read and agreed to the published version of the manuscript.

Funding: This research received no external funding.

Conflicts of Interest: The authors declare no conflict of interest.

\section{Appendix A}

$$
\begin{aligned}
& z_{0}=1 \\
& z_{1}=a_{1}-b_{1} \\
& z_{2}=a_{2}-2 a_{1} b_{1}+2 b_{1}^{2}-b_{2} \\
& z_{3}=a_{3}-3 a_{2} b_{1}+6 a_{1} b_{1}^{2}-6 b_{1}^{3}-3 a_{1} b_{2}+6 b_{1} b_{2}-b_{3} \\
& z_{4}=a_{4}-4 a_{3} b_{1}+12 a_{2} b_{1}^{2}-24 a_{1} b_{1}^{3}+24 b_{1}^{4}-6 a_{2} b_{2}+24 a_{1} b_{1} b_{2}-36 b_{1}^{2} b_{2}+6 b_{2}^{2} \\
& -4 a_{1} b_{3}+8 b_{1} b_{3}-b_{4} \\
& z_{5}=a_{5}-5 a_{4} b_{1}+20 a_{3} b_{1}^{2}-60 a_{2} b_{1}^{3}+120 a_{1} b_{1}^{4}-120 b_{1}^{5}-10 a_{3} b_{2}+60 a_{2} b_{1} b_{2} \\
& -180 a_{1} b_{1}^{2} b_{2}+240 b_{1}^{3} b_{2}+30 a_{1} b_{2}^{2}-90 b_{1} b_{2}^{2}-10 a_{2} b_{3}+40 a_{1} b_{1} b_{3}-60 b_{1}^{2} b_{3} \\
& +20 b_{2} b_{3}-5 a_{1} b_{4}+10 b_{1} b_{4}-b_{5} \\
& z_{6}=a_{6}-6 a_{5} b_{1}+30 a_{4} b_{1}^{2}-120 a_{3} b_{1}^{3}+360 a_{2} b_{1}^{4}-720 a_{1} b_{1}^{5}+720 b_{1}^{6}-15 a_{4} b_{2} \\
& +120 a_{3} b_{1} b_{2}-540 a_{2} b_{1}^{2} b_{2}+1440 a_{1} b_{1}^{3} b_{2}-1800 b_{1}^{4} b_{2}+90 a_{2} b_{2}^{2}-540 a_{1} b_{1} b_{2}^{2} \\
& +1080 b_{1}^{2} b_{2}^{2}-90 b_{2}^{3}-20 a_{3} b_{3}+120 a_{2} b_{1} b_{3}-360 a_{1} b_{1}^{2} b_{3}+480 b_{1}^{3} b_{3}+120 a_{1} b_{2} b_{3} \\
& -360 b_{1} b_{2} b_{3}+20 b_{3}^{2}-15 a_{2} b_{4}+60 a_{1} b_{1} b_{4}-90 b_{1}^{2} b_{4}+30 b_{2} b_{4}-6 a_{1} b_{5}+12 b_{1} b_{5}-b_{6} \\
& z_{7}=a_{7}-7 a_{6} b_{1}+42 a_{5} b_{1}^{2}-210 a_{4} b_{1}^{3}+840 a_{3} b_{1}^{4}-2520 a_{2} b_{1}^{5}+5040 a_{1} b_{1}^{6}-5040 b_{1}^{7} \\
& -21 a_{5} b_{2}+210 a_{4} b_{1} b_{2}-1260 a_{3} b_{1}^{2} b_{2}+5040 a_{2} b_{1}^{3} b_{2}-12600 a_{1} b_{1}^{4} b_{2}+15120 b_{1}^{5} b_{2} \\
& +210 a_{3} b_{2}^{2}-1890 a_{2} b_{1} b_{2}^{2}+7560 a_{1} b_{1} 2 b_{2}^{2}-12600 b_{1}^{3} b_{2}^{2}-630 a_{1} b_{2}^{3}+2520 b_{1} b_{2}^{3}-35 a_{4} b_{3} \\
& +280 a_{3} b_{1} b_{3}-1260 a_{2} b_{1}^{2} b_{3}+3360 a_{1} b_{1}^{3} b_{3}-4200 b_{1}^{4} b_{3}+420 a_{2} b_{2} b_{3}-2520 a_{1} b_{1} b_{2} b_{3} \\
& +5040 b_{1}^{2} b_{2} b_{3}-630 b_{2}^{2} b_{3}+140 a_{1} b_{3}^{2}-420 b_{1} b_{3}^{2}-35 a_{3} b_{4}+210 a_{2} b_{1} b_{4}-630 a_{1} b_{1}^{2} b_{4} \\
& +840 b_{1}^{3} b_{4}+210 a_{1} b_{2} b_{4}-630 b_{1} b_{2} b_{4}+70 b_{3} b_{4}-21 a_{2} b_{5}+84 a_{1} b_{1} b_{5}-126 b_{1}^{2} b_{5} \\
& +42 b_{2} b_{5}-7 a_{1} b_{6}+14 b_{1} b_{6}-b_{7}
\end{aligned}
$$

\section{References}

1. Srivastava, H.M.; Kizilateş, C. A parametric kind of the Fubini-type polynomials. Rev. Real Acad. Cienc. Exactas Fís. Nat. Ser. A Mat. (RACSAM) 2019, 113, 3253-3267. [CrossRef]

2. Srivastava, H.M.; Ricci, P.E.; Natalini, P. A family of complex Appell polynomial sets. Rev. Real Acad. Cienc. Exactas Fís. Nat. Ser. A Mat. (RACSAM) 2019, 113, 2359-2371. [CrossRef]

3. Srivastava, H.M.; Riyasat, M.; Khan, S.; Araci, S.; Acikgoz, M. A new approach to Legendre-truncated-exponential-based Sheffer sequences via Riordan arrays. Appl. Math. Comput. 2020, 369, 124683. [CrossRef]

4. Srivastava, H.M.; Çetinkaya, A.; Kımaz, O. A certain generalized Pochhammer symbol and its applications to hypergeometric functions. Appl. Math. Comput. 2014, 226, 484-491. [CrossRef]

5. Srivastava, R.; Cho, N.E. Generating functions for a certain class of incomplete hypergeometric polynomials. Appl. Math. Comput. 2012, 219, 3219-3225. [CrossRef]

6. Srivastava, R. Some properties of a family of incomplete hypergeometric functions. Russ. J. Math. Phys. 2013, 20, 121-128. [CrossRef]

7. Srivastava, R.; Cho, N.E. Some extended Pochhammer symbols and their applications involving generalized hypergeometric polynomials. Appl. Math. Comput. 2014, 234, 277-285. [CrossRef] 
8. Srivastava, R. Some classes of generating functions associated with a certain family of extended and generalized hypergeometric functions. Appl. Math. Comput. 2014, 243, 132-137. [CrossRef]

9. Srivastava, H.M. Some general families of the Hurwitz-Lerch Zeta functions and their applications: Recent developments and directions for further researches. Proc. Inst. Math. Mech. Nat. Acad. Sci. Azerbaijan 2019, 45, 234-269. [CrossRef]

10. Srivastava, H.M.; Masjed-Jamei, M.; Beyki, M.R. Some new generalizations and applications of the Apostol-Bernoulli, ApostolEuler and Apostol-Genocchi polynomials. Rocky Mt. J. Math. 2019, 49, 681-697. [CrossRef]

11. Lu, D.-Q.; Srivastava, H.M. Some series identities involving the generalized Apostol type and related polynomials. Comput. Math. Appl. 2011, 62, 3591-3602.

12. Lu, D.-Q.; Srivastava, H.M. Some generalizations of the Apostol-Bernoulli and Apostol-Euler polynomials. J. Math. Anal. Appl. 2005, 308, 290-302.

13. Lu, D.-Q.; Srivastava, H.M. Some relationships between the Apostol-Bernoulli and Apostol-Euler polynomials. Comput. Math. Appl. 2006, 51, 631-642.

14. Lu, D.-Q.; Srivastava, H.M. Some generalizations of the Apostol-Genocchi polynomials and the Stirling numbers of the second kind. Appl. Math. Comput. 2011, 217, 5702-5728.

15. Broder, A.Z. The $r$-Stirling numbers. Discret. Math. 1984, 49, 241-259.

16. Connamacher, H.; Dobrosotskaya, J. On the uniformity of the approximation for $r$-associated Stirling numbers of the second kind. Contrib. Discret. Math. 2020, 15, 25-42.

17. Riordan, J. An Introduction to Combinatorial Analysis; J Wiley \& Sons: Chichester, UK, 1958.

18. Carlitz, L. Note on Nörlund's $B_{n}^{(z)}$ Polynomial. Proc. Amer. Math. Soc. 1960, 11, 452-455.

19. Srivastava, H.M.; Todorov, P.G. An Explicit Formula for the Generalized Bernoulli Polynomials. J. Math. Anal. Appl. 1988, 130, 509-513.

20. Srivastava, H.M.; Garg, M.; Choudhary, S. A new generalization of the Bernoulli and related polynomials. Russ. J. Math. Phys. 2010, 17, 251-261.

21. Bretti, G.; Natalini, P.; Ricci, P.E. Generalizations of the Bernoulli and Appel polynomials. Abstract Appl. Anal. 2004, 7, 613-623. [CrossRef]

22. Elezović, N. Generalized Bernoulli Polynomials and Numbers, Revisited. Mediterr. J. Math. 2016, 13, 141-151. [CrossRef]

23. Kurt, B. A Further Generalization of the Bernoulli Polynomials and on the 2D-Bernoulli Polynomials $B_{n}^{2}(x, y)$. Appl. Math. Sci. 2010, 4, 2315-2322.

24. Kurt, B. Some Relationships between the Generalized Apostol-Bernoulli and Apostol-Euler Polynomials. Turk. J. Anal. Number Theory 2013, 1, 54-58. [CrossRef]

25. Miloud, M.; Tiachachat, M. The values of the high order Bernoulli polynomials at integers and the r-Stirling numbers. arXiv 2014, arXiv:1401.5958.

26. Natalini, P.; Bernardini, A. A generalization of the Bernoulli polynomials. J. Appl. Math. 2003, 3, 155-163. [CrossRef]

27. Agarwal, R.P. A propos d'une note de M. Pierre Humbert. C. R. Acad. Sci. Paris 1953, 236, 2031-2032. (In French)

28. Chen, S.; Cai, Y.; Luo, Q.-M. An extension of generalized Apostol-Euler polynomials. Adv. Differ. Equ. 2013, 2013, 61. [CrossRef]

29. Stanley, R.P. Enumerative Combinatorics; Cambridge University Press: Cambridge, UK, 1997.

30. Dattoli, G. Hermite-Bessel and Laguerre-Bessel functions: A by-product of the monomiality principle. In Advanced Special Functions and Applications, Proceedings of the Melfi School on Advanced Topics in Mathematics and Physics, Melfi, Italy, 9-12 May 1999; Cocolicchio, D., Dattoli, G., Srivastava, H.M., Eds.; Aracne Editrice: Rome, Italy, 2000; pp. 147-164.

31. Dattoli, G.; Ricci, P.E.; Srivastava, H.M. (Eds.) Advanced Special Functions and Related Topics in Probability and in Differential Equations. In Applied Mathematics and Computation, Proceedings of the Melfi School on Advanced Topics in Mathematics and Physics, Melfi, Italy, 24-29 June 2001; Aracne Editrice: Rome, Italy, 2003; Volume 141, pp. 1-230.

32. Dattoli, G.; Ricci, P.E.; Cesarano, C. Differential equations for Appell type polynomials. Fract. Calc. Appl. Anal. $2002,5,69-75$.

33. Dattoli, G.; Srivastava, H.M.; Ricci, P.E. Two-index multidimensional Gegenbauer polynomials and integral representations. Math. Comput. Model. 2003, 37, 283-291. [CrossRef]

34. Ben Cheikh, Y. Some results on quasi-monomiality. Appl. Math. Comput. 2003, 141, 63-76. [CrossRef]

35. Comtet, L. Advanced Combinatorics: The Art of Finite and Infinite Expansions; D. Reidel Publishing Co.: Dordrecht, The Netherlands, 1974.

36. Appell, P.; Kampé de Fériet, J. Fonctions Hypergéométriques et Hypersphériques. Polynômes d'Hermite; Gauthier-Villars: Paris, France, 1926.

37. Gould, H.W.; Hopper, A.T. Operational formulas connected with two generalizations of Hermite Polynomials. Duke Math. J. 1962, 29, 51-62. [CrossRef]

38. Srivastava, H.M.; Manocha, H.L. A Treatise on Generating Functions; Halsted Press (Ellis Horwood Limited): Chichester, UK; John Wiley and Sons: Hoboken, NJ, USA, 1984.

39. Dattoli, G.; Lorenzutta, S.; Cesarano, C. Finite Sums and Generalized Forms of Bernoulli Polynomials. Rend. Mat. Ser. VII 1999, 19, 385-391.

40. Natalini, P.; Ricci, P.E. Bell polynomials and some of their Applications. Cubo Mat. Educ. 2003, 5, $263-274$.

41. Natalini, P.; Ricci, P.E. Bell polynomials and modified Bessel functions of half-integral order. Appl. Math. Comput. 2015, 268, 270-274. [CrossRef] 
42. Cassisa, C.; Ricci, P.E. Orthogonal invariants and the Bell polynomials. Rend. Mat. Appl. Ser. VII 2000, $20,293-303$.

43. Qi, F. Three closed forms for convolved Fibonacci numbers. Res. Nonlinear Anal. 2020, 3, 185-195. 